\title{
Wigner-Ville Distribution of a Type of Non-Stationary Mobile Rayleigh Fading Channels
}

\author{
Rauf Iqbal and Thushara Abhayapala \\ $\dagger$ Research School of Information Sciences and Engineering \\ Australian National University \\ Canberra, Australia \\ Email: FirstName.LastName@anu.edu.au
}

\author{
Javed Ahmed \\ Military College of Signals \\ NUST, Rawalpindi, Pakistan \\ Email: javed@mcs.edu.pk
}

Tharaka Lamahewa $†$

\begin{abstract}
The classical Clarke model of mobile radio reception assumes a constant mobile velocity. We, in this paper, relax the assumption of constant mobile velocity to allow the mobile to have constant acceleration and derive expression for the non-stationary autocorrelation function of the channel process in general 2-dimensional (2D) scattering environments. Under suitable assumptions, an expression for Wigner-Ville spectrum is obtained in isotropic scattering environment which suggests that the Wigner-Ville spectrum is a natural generalization of the Clarke's model to constant mobile acceleration scenario.
\end{abstract}

\section{INTRODUCTION}

The Clarke's model of mobile radio reception [1] is frequently used in design and analysis of wireless communications systems. The channel between a stationary transmitter and a mobile receiver equipped with omnidirectional antenna is modeled as a stationary complex Gaussian process based on the assumptions that the scattering around the mobile receiver is statistically homogeneous, the magnitudes and phases of the arriving waves are independent, the distribution of angles of arrival (AOA) over $[-\pi, \pi]$ is uniform and the mobile moves with constant velocity. Furthermore, all these assumptions remain true irrespective of absolute time.

Several generalizations of the Clarke's model exist in literature, e.g., [2-6]. However, all these generalizations assume a constant mobile velocity or, equivalently, stationary channel statistics. In this contribution we present a generalization of the Clarke's model by relaxing the assumption of constant mobile velocity to allow the mobile receiver to have constant acceleration in a general scattering environment. The mobile motion with constant acceleration corresponds better to the physical reality because a mobile user may experience changes in velocity caused by traffic lights or road conditions [7]. Moreover, the emerging wireless communications standards specify higher carrier frequencies ${ }^{1}$ which are intrinsically more sensitive to mobile motion. The impact of mobile acceleration in such communications systems may not be ignorable and the assumption of constant mobile velocity may not be justified.

\footnotetext{
${ }^{1}$ The IEEE $802.16 \mathrm{e}$ standard also known as mobile Worldwide Interoperability for Microwave Access (WiMax) specifies mobile communications at carrier frequencies from $2-11 \mathrm{GHz}$ in non-line-of-sight (NLOS) environments $[8,9]$.
}

\section{A. Channel Non-Stationarity due to Constant Mobile Accel- eration}

For a fixed carrier frequency $f_{c}$, the Doppler spectrum of the channel fading process depends directly on the mobile velocity. We have the following relationship between a particular mobile velocity $\boldsymbol{v}$ and a particular Doppler frequency $f_{d}$ present in the Doppler spectrum

$$
f_{d}=\frac{|\boldsymbol{v}|}{\lambda_{c}} \cos \gamma,
$$

where $\lambda_{c}=c / f_{c}$ is the carrier wavelength ( $c$ is the speed of light), and $\gamma$ is the angle between the scattering angle and the unit vector $\hat{\boldsymbol{v}}$ (pointing in the direction of mobile movement). The maximum Doppler frequency, $f_{d}^{\max }=|\boldsymbol{v}| / \lambda_{c}$ corresponds to the wave impinging on the mobile receiver head-on. When the mobile receiver has constant acceleration $\alpha$, the velocity of the mobile changes continuously with time. If, for example, $\boldsymbol{v}^{\prime}$ denotes the mobile velocity at some time $t^{\prime}$, the velocity of the mobile at time $t>t^{\prime}$ is given by

$$
\boldsymbol{v}(t)=\boldsymbol{v}^{\prime}+\alpha\left(t-t^{\prime}\right)
$$

The time-varying velocity would imply a time-varying power spectral density (PSD) and, hence, autocorrelation function (ACF). Equivalently, the channel would become a nonstationary complex Gaussian process as a result of constant mobile acceleration and the estimation of the spectral contents of the non-stationary fading process based on stationarity assumption would be inaccurate [10-12]. When a signal or a random process has time-varying characteristics, a timefrequency representation - a generalization of the classical Fourier transform which transforms the signal (or random process) in time-domain to time-frequency plane - is most suitable [10].

\section{B. Short background and Motivation}

Recently, the impact of non-stationarity due to constant mobile acceleration has been discussed in [13] in relation to correlation based speed estimation for indoor positioning where the constant mobile acceleration, if ignored, was found to introduce errors in speed estimation. The impact of mobile acceleration on linear prediction (and subsequent equalization) of the envelope of frequency-flat complex Gaussian fading 
was investigated in [14]. The constant mobile acceleration was shown to have an impact on the choice of the adaptive prediction algorithm. For example, the linear prediction based on windowed recursive least squares (WRLS) algorithm performed better than the recursive least squares (RLS) algorithm for a mobile with constant acceleration. These studies of the mobile acceleration impact have been based simply on simulations. To the best of our knowledge, the wireless channel statistics and the performance of a cellular wireless mobile communication system with accelerating mobile receiver have not been analyzed in the literature as yet, perhaps, because the state-of-the-art wireless communication systems operate at frequencies of the order of $2 \mathrm{GHz}$ having sufficiently short transmission frame lengths. Moreover, the channel is estimated for each frame independently of other frames. The impact of mobile acceleration on the estimation of parameters of interest such as channel gain and maximum Doppler frequency for each frame is not significant over the duration of the frame due to mass inertia effect and, therefore, can be ignored without significantly losing accuracy for all practical acceleration possibilities for mobile speeds as high as $\approx 200 \mathrm{~km} / \mathrm{hr}$. In this contribution, under suitable assumptions, we analytically show that Wigner-Ville distribution, one of the most popular timefrequency energy distributions, extends the Clarke's stationary Rayleigh fading model to non-stationary fading due to constant mobile acceleration. The results may be important for the design and performance evaluation of a particular aspect of a communications system involving an accelerating mobile receiver or its performance as a whole.

This paper is organized as follows. The Section II describes the continuous time and discrete time channel models and specifies different assumptions about various parameters involved. The Section III contains a brief introduction of the . The continuous-time and discrete-time instantaneous channel autocorrelation and corresponding expression for Wigner-Ville distribution are derived in Section IV based on assumption that the mobile velocity can be unbounded and we have infinite sampling rate capability at our disposal. The implications of the assumption of bounded mobile velocity and finite sampling rate are also discussed. Finally, the conclusions are drawn in Section V.

\section{Channel Model}

We consider a downlink mobile communications scenario in which the transmitter is stationary while, at time $t$, the receiver equipped with an omni-directional antenna moving with initial velocity $\boldsymbol{v}$ and constant acceleration ${ }^{2} \alpha$ at angle $\psi$ with respect to $\mathrm{x}$-axis in a sufficiently rich $2 \mathrm{D}$ general scattering environment ${ }^{3}$ [15] (Fig. 1).

\footnotetext{
${ }^{2} \mathrm{We}$ assume that the mobile acceleration is aligned with velocity. The term 'acceleration' may, therefore, be interpreted as the magnitude of the acceleration.

${ }^{3}$ The scatterers are assumed to be located on a circle in the far field so that the waves impingent on the receive antenna can be approximated as electromagnetic plane waves.
}

If $s(t)$ is the continuous-time signal transmitted at time $t$, the received signal $r(t)$ is given in baseband form as follows:

$$
r(t)=\sqrt{\rho} h_{c}(t) s(t)+z(t) \quad-\infty<t<\infty,
$$

where $h_{c}(t)$ is the non-stationary continuous-time complex channel process and $z(t)$ is the additive white Gaussian noise (AWGN). It is also assumed that the input, channel and noise processes are independent processes. We also assume that the transmitted signal, channel process and the AWGN noise are of unit variance so that $\rho$ represents the signal-to-noise ratio (SNR). The accelerating mobile moving with initial velocity $\boldsymbol{v}_{0}$ is assumed to be at some arbitrary origin ' $\mathrm{O}$ ' at $t=0$. The continuous-time non-stationary channel process $h_{c}(t)$

$$
h_{c}(t)=\oint \psi(\beta) \exp (i \eta t \boldsymbol{v}(t) \cdot \hat{\boldsymbol{\beta}}) d \beta,
$$

where $\boldsymbol{v}(t)$ is the instantaneous velocity, $\eta=2 \pi / \lambda$ is the plane wave phase constant ( $\lambda$ represents the carrier wavelength) and $\oint$ represents integration over a circle. If we further assume that the scattering is zero-mean statistically homogeneous, the magnitudes and phases of the impingent waves are independent and the phases are uniformly distributed over $[-\pi, \pi]$, the channel process $h_{c}(t)$ is a zero-mean complex Gaussian process with independent real and imaginary parts of variance $1 / 2$. Equivalently, the envelope of the channel is Rayleigh distributed.

\section{A. Discretization of Continuous-Time Channel Process}

We assume that the instantaneous sampling rate is at least twice the instantaneous Doppler frequency which admits $t$ following discrete-time equivalent of the continuous-time inputoutput relationship

$$
r[j]=\sqrt{\rho} h[j] s[j]+z[j], \quad-\infty<j<\infty
$$

where the discrete-time input, output, channel and noise processes retain the statistical properties of their continuous-time counterparts. It may be noticed that we have dropped the subscript $d$ for notational simplicity. In the Clarke's stationary Rayleigh fading channel model and generalizations thereof [26], the channel spectrum is time-invariant making it possible to have a fixed sampling rate. In the non-stationary scenario at hand, we are at freedom to either fix or use variable transmission and sampling rates to ensure, at least, Nyquist sampling of the channel process.

1) Fixed Transmission and Sampling Rates: If the communication system designer can foresee the maximum time for which the mobile keeps on moving with the constant acceleration or, equivalently, the maximum Doppler spread is known at the transmitter a priori, it is possible to fix the transmission rate such that the channel is sampled at least at the Nyquist rate in worst channel conditions. As an example, let us assume that the maximum Doppler frequency is $200 \mathrm{~Hz}$. The Nyquist rate corresponding to this rate of channel variation equals 400 $\mathrm{Hz}$ which implies that the channel samples required at the receiver to form CSI estimate must be furnished at least at the rate of 400 samples per second. This sampling rate, in turn, 


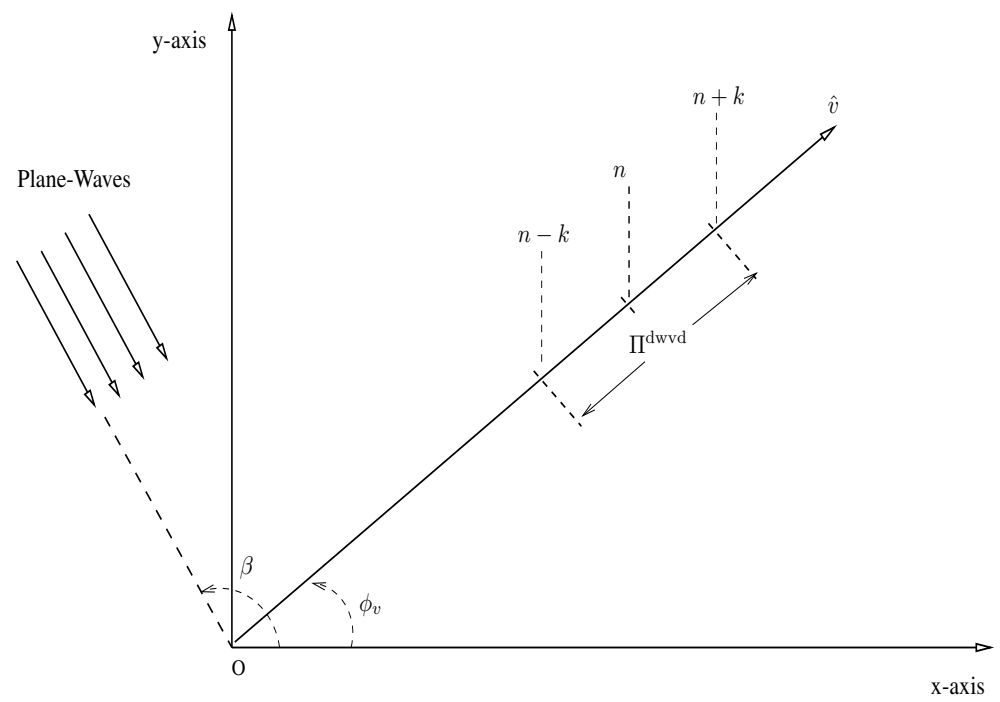

Fig. 1. The mobile is moving at an angle $\phi_{v}$ with respect to $\mathrm{x}$-axis with initial speed $\left|\boldsymbol{v}_{0}\right|$ and constant acceleration $\alpha$ in the direction of movement. A plane wave is shown incident on the receive antenna at an angle $\beta$ and $\Pi^{d w v d}$ defined in (24) is also depicted.

requires the symbol duration to be $2.5 \mathrm{msec}$. Since the symbol rate is fixed and the channel has to be sampled at symbol rate, at a time when the maximum Doppler frequency is $100 \mathrm{~Hz}$, the channel would get oversampled at twice the Nyquist rate. In fact, when the transmission rate is fixed according to worst channel conditions, the channel gets oversampled for almost the full duration of transmission. Therefore, the cost that has to be paid for keeping the transmission rate fixed is the added complexity due to oversampling of the channel process.

2) Adaptive Transmission and Sampling Rates: If $T_{s}^{\prime}$ is the sampling period, the channel fading rate normalized to the symbol rate, $f_{\mathrm{D}}$, is given as (equation (1))

$$
f_{\mathrm{D}}=f_{d} T_{s}^{\prime},
$$

which implies that the normalized fading rate can be forced to be constant if we can change $T_{s}^{\prime}$ (equivalently, the symbol rate at the transmitter). In other words, the transmission scheme should adapt to the instantaneous Doppler spread which, however, requires a priori knowledge of the instantaneous channel spectrum at the transmitter. The receiver must also have the exact knowledge of the transmission rate implying more stringent adaptive synchronization requirements at the receiver.

\section{Wigner-VILLE DisTRIBUTION (WVD)}

\section{A. Continuous-Time WVD}

The WVD (a member of Cohen's class [16] - a class of quadratic time-frequency energy distributions) is one of the most widely used TF methods [17]. This approach was developed by Wigner [18] in 1932 for use in Physics. Later Ville [19] introduced this technique to signal processing community in 1948. The classical method of determining the spectral contents of a random process is based on the well-known Wiener Khintchine theorem, i.e., the spectrum of a random process equals the Fourier transform of the classical ACF which is a function of the lag variable, $\tau$, only. The classical ACF is time-invariant because the time is integrated out of the result. The WVD is based on a variation of the classical ACF so that it retains time variable in the result. Such ACF is termed as instantaneous ACF, $\left(\Phi_{s}^{w v d}(t, \tau)\right)_{\text {inst }}$. Assuming a deterministic signal $s(t)$, continuous-time instantaneous ACF is given as

$$
\left(\Phi_{s}^{w v d}(t, \tau)\right)_{\text {inst }}=s\left(t+\frac{\tau}{2}\right) s^{*}\left(t-\frac{\tau}{2}\right),
$$

and corresponding time-frequency energy distribution is defined as

$$
\begin{aligned}
\mathcal{E}^{w v d}(t, f) & \triangleq \operatorname{WVD}(t, f), \\
& =\int_{-\infty}^{\infty}\left(\Phi_{s}^{w v d}(t, \tau)\right)_{i n s t} \exp (-i 2 \pi f \tau) d \tau .
\end{aligned}
$$

The WVD is, in fact, a distribution of the mean instantaneous power versus frequency, or equivalently, a distribution of the mean energy in the joint time-frequency plane [12]. The WVD satisfies marginals but can be negative. However, the Gaussian smoothing implemented by convolving a Gaussian function with instantaneous ACF in (7), can ensure that it is always positive. Importantly, the smoothing makes WVD identical to a spectrogram [20] with reduced cross-term interference when multiple components are present in the signal, e.g., a mobile radio signal. The smoothing operation in time results in a smoothed WVD, the smoothing in frequency results in pseudo-WVD and the smoothing in both time and frequency would result in smoothed pseudo-WVD [21,22].

Equation (8) is valid for deterministic signal $s(t$ ) (or a random process with only a single realization). Since we are dealing with a random channel process, we need to have a definition of WVD for random process with known statistics. The WVD for the random channel process $h_{c}(t)$ is defined as the expectation of $\operatorname{WVD}(t, f)$ (equation (8)) over all possible 
realizations implied by the assumed channel statistics, i.e.,

$$
\begin{aligned}
\operatorname{WVD}(t, f) & =\mathbb{E}\left\{\int_{-\infty}^{\infty}\left(\Phi_{h}^{w v d}(t, \tau)\right)_{i n s t} \exp (-i 2 \pi f \tau) d \tau\right\} \\
& =\int_{-\infty}^{\infty} \Phi_{h}^{w v d}(t, \tau) \exp (-2 \pi f \tau) d \tau
\end{aligned}
$$

where we have defined

$$
\Phi_{h}^{w v d}(t, \tau)=\mathbb{E}\left\{\left(\Phi_{s}^{w v d}(t, \tau)\right)_{\text {inst }}\right\} .
$$

The interchange of the expectation and the integral in (9) is justified if the integral exists in the mean-square sense for which a necessary and sufficient condition is [23]

$$
\int_{-\infty}^{\infty} \int_{-\infty}^{\infty} \mathbb{E}\left\{q_{h}\left(t, \tau_{2}\right) q_{h}^{*}\left(t, \tau_{2}\right)\right\} e^{-i 2 \pi f\left(\tau_{1}-\tau_{2}\right)} d \tau_{1} d \tau_{2}<\infty .
$$

\section{B. Discrete-Time WVD}

The continuous-time WVD (equation (9)) has the following logical extension to the discrete-time domain

$$
\operatorname{DWVD}\left(n T_{s}^{\prime}, f\right)=2 \sum_{k=-\infty}^{\infty} \Phi_{h}^{\prime}(n, k) \exp \left(-i 2 \pi f k T_{s}^{\prime}\right),
$$

where DWVD represents discrete-time WVD, $T_{s}^{\prime}$ is the Nyquist sampling period and $\Phi_{h}^{\prime}(n, k)$ is the discrete-time equivalent of the continuous-time $\mathrm{ACF}, \Phi_{h}^{\prime}(t, k)$, given as

$$
\Phi_{h}^{\prime}(n, k)=\mathbb{E}\left\{h\left(n-\frac{k}{2}\right) h^{*}\left(n-\frac{k}{2}\right)\right\} .
$$

It may be noticed that (12) involves lags at infinity which implies that the mobile with constant acceleration needs to have unbounded velocity in order to admit description in the frequency domain in the Fourier sense! In this paper, we make the simplifying assumptions of unbounded mobile velocity and infinite sampling rate (required theoretically for proper discretization of the channel process). We shall discuss these assumptions in more detail in Section IV.

Notice that (12) suffers from aliasing (as we have assumed Nyquist rate sampling) and requires samples of the channel process at non-integer instants which are not available if the channel process is sampled at the symbol rate. A number of different methods have been proposed in literature to overcome this problem [24]. We shall, however, follow the technique based on oversampling assuming that the channel process is sampled at least at twice the Nyquist rate. This assumption enables us to write (12) as follows [25]

$$
\operatorname{DWVD}\left(n T_{s}, f\right)=2 \sum_{k=-\infty}^{\infty} \Phi_{h}^{d w v d}(n, k) \exp \left(-i 2 \pi f k T_{s}\right)
$$

where $T_{s}=T_{s}^{\prime} / 2$ is the sampling period corresponding to twice the Nyquist sampling rate ${ }^{4}$

$$
\Phi_{h}^{d w v d}(n, k)=\mathbb{E}\left\{h(n+k) h^{*}(n-k)\right\} .
$$

\footnotetext{
${ }^{4}$ The assumption of twice the Nyquist rate sampling simplifies the following analysis.
}

Equation (15) gives DWVD for a general channel autocorrelation function $\Phi_{h}^{d w v d}(n, k)$.

\section{Formulation of Instantaneous Channel ACF AND DWVD}

Let the mobile moving with initial velocity $\boldsymbol{v}_{0}=\left|\boldsymbol{v}_{0}\right|, \phi_{v}$ and acceleration $\alpha$ be at an arbitrary origin $O$ at the signaling instant 0 (Fig. 1). We can see from equation (15) that the formulation of ACFs for DWVD and DMIPS would require the determination of channel gains at instants $n, n-k$ and $n+k$ where $k$ is the lag in number of symbols. At the signaling interval $n$, let the mobile be at the point $\left(\Pi_{n}, \phi_{v}\right)$ with respect to signaling instant 0 , where $\phi_{v}$ is the direction of the mobile with respect to $\mathrm{x}$-axis. At the signaling interval $n-k$, the mobile is assumed to be at point $\left(\Pi_{n-k}, \phi_{v}\right)$. Similarly, at the signaling instant $n+k$, the mobile is assumed to be at $\left(\Pi_{n+k}, \phi_{v}\right)$. Using the fact that the mobile is accelerating in the direction of mobile travel, we have the following relationships for distances covered by the mobile

$$
\begin{aligned}
& \left.\Pi_{n-k}=\left|\boldsymbol{v}_{0}\right|(n-k) T_{s}+\frac{1}{2} \alpha\left((n-k) T_{s}\right)\right)^{2}, \\
& \left.\Pi_{n+k}=\left|\boldsymbol{v}_{0}\right|(n+k) T_{s}+\frac{1}{2} \alpha\left((n+k) T_{s}\right)\right)^{2},
\end{aligned}
$$

and corresponding channel gains are

$$
\begin{aligned}
& h[n-k]=\oint \psi(\beta) \exp \left(i \eta \Pi_{n-k} \hat{\boldsymbol{v}} \cdot \hat{\boldsymbol{\beta}}\right) d \beta, \\
& h[n+k]=\oint \psi(\beta) \exp \left(i \eta \Pi_{n+k} \hat{\boldsymbol{v}} \cdot \hat{\boldsymbol{\beta}}\right) d \beta .
\end{aligned}
$$

Now that we have instantaneous channel gains, using (19) and (20), the instantaneous ACF for DWVD is given as (equation (15))

$$
\begin{aligned}
\Phi_{h}^{d w v d}(n, k) & =\mathbb{E}\left\{h(n+k) h^{*}(n-k)\right\}, \\
& =\oint \Psi(\beta) \exp \left(i \eta \Pi^{d w v d} \hat{\boldsymbol{v}} \cdot \hat{\boldsymbol{\beta}}\right) d \beta,
\end{aligned}
$$

where (22) is the result of the assumption of statistically homogeneous scattering, $\Psi(\beta)$ is the azimuth power distribution (APD) [15] defined as

$$
\Psi(\beta)=\mathbb{E}\left\{\psi(\beta) \psi^{*}\left(\beta^{\prime}\right)\right\} u\left(\beta-\beta^{\prime}\right),
$$

where $u(\cdot)$ is the unit step function. The factor $\Pi^{d w v d}$ in (22) defined as

$$
\begin{aligned}
\Pi^{d w v d} & =\left(\Pi_{n+k}-\Pi_{n-k}\right), \\
& =2 k T_{s}\left(\left|\boldsymbol{v}_{0}\right|+\alpha\left(n T_{s}\right)\right),
\end{aligned}
$$

is the distance covered by the mobile at signaling instant $n+k$ with respect to the signaling instant $n-k$ (Fig. 1).

Equation (22) involves the product of two periodic functions, $\Psi(\beta)$ and $\exp \left(i \eta \Pi^{x} \hat{\boldsymbol{v}} \cdot \hat{\boldsymbol{\beta}}\right)$. The representation of these periodic functions by their respective Fourier series would result in their Fourier series coefficients to be convoluted 


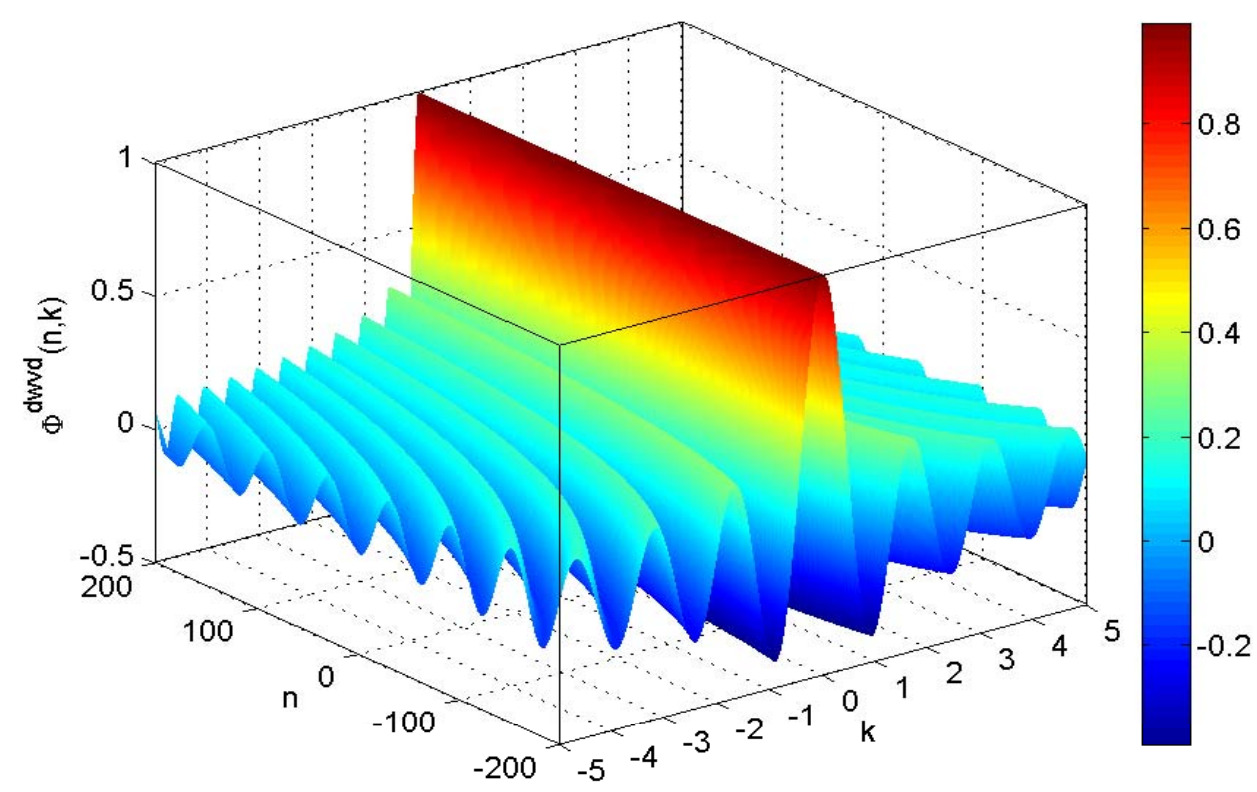

Fig. 2. The ACF of DWVD as a function of the lag $k$ and absolute time index $n:\left|\boldsymbol{v}_{0}\right|=20 \mathrm{~m} / \mathrm{sec}, \alpha=10 \mathrm{~m} / \mathrm{sec}^{2}, f_{c}=2 \mathrm{GHz}$ and $T_{s}=5 \mathrm{msec}$. Increasing $n$ and $k$ have the effect of reducing the autocorrelation faster.

[15]. By following the approach adopted in [15], the ACF for DWVD can be written as

$$
\Phi_{h}^{d w v d}(n, k)=\sum_{m=-\infty}^{\infty} i^{m} \gamma_{m} J_{m}\left(\eta \Pi^{w v d}\right) \exp \left(i m \phi_{v}\right)
$$

where $\gamma_{m}$ are the coefficients of the Fourier series expansion of $\Psi(\beta)$ given as

$$
\gamma_{m}=\oint \Psi(\beta) e^{-i m \beta} d \beta
$$

and $J_{m}(\cdot)$ is the Bessel function of the first kind of order zero which appears as a result of the Jacobi-Anger expansion [26] of the plane wave $\exp \left(i \eta \Pi^{d w v d} \hat{\boldsymbol{v}} \cdot \hat{\boldsymbol{\beta}}\right)$. Equation (27) defines ACF for DWVD in general scattering environments. To keep things simple, we assume that the scattering environment around the mobile receiver is isotropic so that (27) can be rewritten as

$$
\Phi_{h}^{d w v d}(n, k)=J_{0}\left(\eta \Pi^{w v d}\right) .
$$

We have plotted $\Phi_{h}^{d w v d}(n, k)$ in Fig. 2 showing non-stationary character of the ACF.

The discrete-time WVD in an isotropic scattering environment is defined as

$$
\begin{aligned}
& \operatorname{DWVD}_{i s o}\left(n T_{s}, f\right) \\
& =\frac{1}{T_{s}} \sum_{k} \Phi_{h}^{d w v d}(n, k) \exp \left(-i \omega k T_{s}\right) \\
& ,=\frac{1}{T_{s}} \sum_{k} J_{0}(\overbrace{2 \omega_{D, 0}\left(1+\alpha^{N} n\right)}^{\hat{\omega}_{D, n}} k) \exp \left(-i \omega k T_{s}\right),
\end{aligned}
$$

where $\omega=2 \pi f(f$ is the frequency in hertz $(\mathrm{Hz}))$ and $\widehat{\omega}_{D, n}$ is the instantaneous normalized Doppler spread for DWVD spectrum. Mathematically,

$$
\begin{aligned}
\widehat{\omega}_{D, n} & =2 \omega_{D, 0}\left(1+\alpha^{N} n\right), \\
& =2 \frac{\left|\boldsymbol{v}_{0}\right| T_{s}}{\lambda_{c}}\left(1+\frac{\alpha n T_{s}}{\left|\boldsymbol{v}_{0}\right|}\right)
\end{aligned}
$$

Let us consider the following DTFT relationship for the Bessel function of the first kind of integer order $\mu[15,27]$

$$
\sum_{\ell=-\infty}^{\infty} J_{\mu}\left(\widehat{\omega}_{D, n} \ell\right) \exp \left(-i \omega \ell T_{s}\right)=\frac{F_{\mu}\left(\omega / \widehat{\omega}_{D, n}\right)}{i^{\mu} \widehat{\omega}_{D, n}},
$$

where $F_{\mu}$ is given as

$$
F_{\mu}(x) \triangleq 2 \frac{\cos \left(\mu \cos ^{-1}(x)\right)}{\sqrt{1-x^{2}}} .
$$

Using (29) and (32) and discarding all terms except corresponding to $\mu=0$, we can write DWVD in isotropic scattering environment as

$$
\operatorname{DWVD}\left(n T_{s}, f\right)=\frac{2 \widehat{\omega}_{D, n}}{\sqrt{\widehat{\omega}_{D, n}^{2}-\omega^{2}}}, \text {, }
$$

which is true for $\omega \leq \widehat{\omega}_{D, n}$. Since $\widehat{\omega}_{D, n}>0$, DWVD in equation (34) is always non-negative. An important implication of (34) is that, for a finite $n$, the channel process has a bandlimited representation. Equation (34) represents the generalization of the Clarke's model from stationary to nonstationary case which is what we set out to show. 


\section{REMARKS:}

The derivation of DWVD spectrum (equation (34)) implicitly assumes that the lag $k$ is allowed to approach infinity as required by the definition of DTFT. By allowing $k$ to approach infinity for any $n<\infty$, we are in fact assuming that the transmission frame is of infinite length and the mobile velocity is allowed to increase without bound becoming infinite when $k$ is infinity. Moreover, the discretization of the continuoustime Bessel function of the first kind of order zero with linear argument without aliasing requires infinite sampling rate [28]. The assumption of infinite sampling rate is obviously a convenient mathematical construct rather than a physical reality in view of the fact that nothing can move faster than the speed of the light [29] and only finite sampling rate is possible. To be more relevant, the maximum speed of vehicles is only a few hundred $\mathrm{km} / \mathrm{hr}$, i.e., only a very slight fraction of the speed of the light and a vehicle can keep on accelerating only for a finite time. Both of these real-world facts imply that neither $n$ nor $k$ can approach infinity. The finiteness of $n$ and $k$ would require finite-length windows in the time $n$ and lag $k$ domains in the DTFT representation defined in (32). Our assumption of the availability of infinite sampling rate removes the need for an analog prefilter before analog-todigital converter (ADC) used to limit the bandwidth of infinitebandwidth AWGN noise. A consequence of infinite sampling rate is that the discrete-time AWGN in (5) is truly white.

\section{Conclusions}

We considered a downlink communications scenario in which the mobile receiver moves with constant acceleration through sufficiently rich 2D general scattering environment. The electromagnetic plane-wave theory was applied to arrive at expression for non-stationary autocorrelation function of the channel process in general scattering environments. Assuming that the mobile receiver could have unbounded velocity and infinite sampling rate is possible, we derived an expression for the Wigner-Ville spectrum in an isotropic scattering environment which happens to be a natural generalization of the Clarke's model from constant mobile velocity (stationary) to constant mobile acceleration (non-stationary) scenario. The results can be used for the performance analysis of communications systems and can be considered as a benchmark for real-world scenarios where only a finite data record may be available for spectral characterization.

\section{REFERENCES}

[1] R. H. Clarke, "A statistical theory of mobile-radio reception," Bell Systems Technical Journal, vol. 47, no. 6, pp. 957-1000, 1968.

[2] J. T. Y. Ho, "Generalized doppler power spectrum for 3D non-isotropic scattering environments," in IEEE Global Telecommunications Conference, GLOBECOM'05, vol. 3, 2005, p. 13931396.

[3] A. Abdi, J. A. Barger, and M. Kaveh, "A parametric model for the distribution of the angle of arrival and the associated correlation function and power spectrum at the mobile station," vol. 51, no. 3, pp. 425-434, May 2002.

[4] K. Anim-Appiah, "Complex envelope correlations for non-isotropic scattering," IEE Electronics Letters, vol. 34, no. 9, pp. 1039-1052, Apr 1998.
[5] H. S. Rad and S. Gazor, "A cross-correlation MIMO channel model for non-isotropic scattering environment and non-omnidirectional antennas," in Candian Conference on Electrical and Computer Engineering, 2005. May 2005, pp. 25-28.

[6] J. S. Sadowsky and V. Katedziski, "On the correlation and scattering functions of the WSSUS channel for mobile communications," IEEE Transactions on Vehicular Technology, vol. 47, no. 1, pp. 270-282, Feb. 1998.

[7] T. C. Jeff, J. Boleng, and V. Davies, "A survey of mobility models for ad hoc network research," Wireless Communications \& Mobile Computing (WCMC): Special issue on Mobile Ad Hoc Networking: Research, Trends and Applications, vol. 2, pp. 483-502, 2002.

[8] Y. Zhang and H.Chen, Eds., Mobile WiMAX: toward broadband wireless metropolitan area networks. CRC Press, 2007.

[9] D. S. Holder, Ed., Electrical Impedance Tomography: Methods, History and Applications. Bristol and Philadelphia: Institute of Physics, 2005.

[10] S. Qian and D. Chen, "Joint time-frequency analysis," IEEE Sig. Proc. Mag., pp. 53-67, Mar. 1999.

[11] W. Martin and P. Flandrin, "Wigner-Ville spectral analysis of nonstationary processes," Proc. IEEE Int. Conf. Acoust., Speech Signal Processing, vol. ASSP-33, pp. 1461-1470, Dec. 1985.

[12] F. Hlawatsch and W. Kozek, "Second-order time-frequency synthesis of nonstationary random processes," IEEE Trans. Info. Theory, vol. 41, no. 1 , pp. 1461-1470, Jan. 1995

[13] M. Afgani, S. Sinanović, K. Kashaba, and H. Haas, "Radio frequency signature correlation based speed estimation for indoor positioning," Journal of Communications, vol. 4, no. 2, pp. 96-107, Mar. 2009.

[14] P. Roy and A. A. Beex, "Flat fading channel envelope prediction and equalization," in Proc. Thirty-Fifth IEEE Asilomar Conference on Signals, Systems and Computers, vol. 2, Pacific Grove, CA, USA, 2001, pp. 1116-1120.

[15] R. Iqbal, T. Abhayapala, and T. Lamahewa, "Generalized Clarke model for mobile radio reception," IET Communications, vol. 3 , no. 4, pp. 644-654.

[16] L. Cohen, "Time frequency distributions- a review," Proceedings of IEEE, vol. 77, no. 7, pp. 941-981, 1989.

[17] G. Matz and F. Hlawatsch, "Wigner distributions (nearly) everywhere: time-frequency analysis of signals, systems, random processes, signal spaces, and frames," Signal Process., vol. 83, no. 7, pp. 1355-1378, 2003.

[18] E. P. Wigner, "On the quantum correction for thermodynamic equilibrium," Physical Review, vol. 40, p. 749, Mar. 1932.

[19] J. Ville, "theorie et applications de la notion de signal analytique," $C$ \& T, vol. 2-A, no. 1, 1948.

[20] A. P. Suppappola, Ed., Applications in time-frequency signal processing. CRC Press, 2002

[21] L. M. Khadra, "The smoothed pseudo-Wigner distribution in speech processing," International Journal of Electronics, vol. 65, no. 4, pp. 743-755, 1988.

[22] T. A. C. M. Claasen and W. F. G. Mecklenbräuker, "The Wigner distribution-a tool for time-frequency signal analysis, part i. continuoustime signals," Philips J. Res., vol. 35, pp. 217-250, 1980.

[23] A. M. Sayeed and D. L. Jones, "Optimal kernels for nonstationary spectral estimation," IEEE Trans. on Signal Processing, vol. 43, no. 2, pp. 478-491, Feb. 1995.

[24] S. S. Abeysekera, "computation of Wigner-Ville distribution for complex data," IEEE Elect. Lett., vol. 26, no. 16, pp. 1315-1317, Aug. 1990.

[25] T. A. C. M. Claasen and W. F. G. Mecklenbräuker, "The Wigner distribution-a tool for time-frequency signal analysis, part i. continuoustime signals," Philips J. Res., vol. 35, no. 4/5, pp. 276-300, 1980.

[26] D. Colton and R. Kress, Inverse Acoustic and Electromagnetic Scattering Theory, 2nd ed. New York: Springer, 1998, vol. 93.

[27] A. Y. Rom, "Ground state of a spinless charged particle in a periodic magnetic field," Physical Review B, vol. 55, no. 17, pp. 11025-11028, May 1997.

[28] L. Deng and D. O'Shaughnessy, Speech processing: a dynamic and optimization-oriented approach. CRC Press, 2003.

[29] P. W. Atkins, Galileo's finger: the ten great ideas of science. Oxford University Press, 2003. 